\title{
An Automated Music Recommendation System Based on Listener Preferences
}

\author{
Mukkamala. S.N.V. Jitendra ${ }^{\mathrm{a}, 1}, \mathrm{Y}$. Radhika ${ }^{\mathrm{a}}$ \\ ${ }^{a}$ Department of Computer Science and Engineering, GIT, GITAM (Deemed-to-be \\ University), Visakhapatnam-530045, AP, India
}

\begin{abstract}
Recommender systems play a vital role in e-commerce. It is a big source of a market that brings people from all over the world to a single place. It has become easy to access and reach the market while sitting anywhere. Recommender systems do a major role in the commerce mobility go smoothly easily as it is a software tool that helps in showing or recommending items based on user's preferences by analyzing their taste. In this paper, we make a recommender system that would be specifically for music applications. Different people listen to different types of music, so we make note of their taste in music and suggest to them the next song based on their previous choice. This is achieved by using a popularity algorithm, classification, and collaborative filtering. Finally, we make a comparison of the built system for its effectiveness with different evaluation metrics.
\end{abstract}

Keywords. E-commerce, recommender system, collaborative filtering, user-based recommendation, item-based recommendation, music information retrieval.

\section{Introduction}

On the web, where the measure of decisions is overpowering, there's a prerequisite to filter, organize and proficiently convey significant data to lighten the matter of data overload, which has made a potential issue for a few Internet users. Recommendation settles the issue of giving the best outcomes by giving the results of top information which is dynamically generated using the required methods and data.

Moreover, of late, interpersonal organizations turned out to be generally utilized and well-known modes for information dissemination likewise on account of the facilitators of social interaction. User commitment and exercises give important understanding into singular conduct, encounters, assessments, and interests. Considering the metadata together with the client information gives more approaches to build the exhibition of strategies like shared separating. Recommendation systems have been introduced into a variety of areas with challenges. These include Government recommendation systems, Government service recommendation, E-business recommendation systems, A Telecom recommendation system, Commerce/shopping recommendation systems, Library recommendation systems, Learning recommendation systems, Tourism recommendation systems, Service recommendation systems [1].

\footnotetext{
${ }^{1}$ Mukkamala.S.N.V. Jitendra, Department of Computer Science and Engineering, GITAM, India 
Various models used models for music recommendation can including music recommendation models such as content-based, emotional, which is used for people; collaborative, which uses information in addition to the content; and additional content; and metadata-based, which includes information about music and genres [2]. The collaborative recommendation has served well but has weaknesses such as being prone to popularity bias, the limitations of human actions, etc. Concerning the other disadvantages, the hybrid system has not yet been studied extensively, but on the other hand, it does offer greater performance concerning the breadth of opinion. Both the emotional and social information are considered models of the recommendation that have a great impact on the overall quality of the recommendations This topic is just beginning to be researched and will continue to expand in the coming years.

Today almost every e-commerce website or online streaming site uses the Recommender System to make it easier for the users to access and profitable on both ends. The sites like Netflix, Amazon, Flipkart, etc. generally use these to maintain their users by providing suggestions that will be mostly liked by them. The Recommenders use the user's search history and other statistics to recommend the products. A famous example of such a recommendation is the one with amazon. It suggests the products similar to the product being viewed and also the products which the other customers bought and viewed the same product. Similarly, Netflix does the recommendations based on the type of movie watched, language, cast, theme, or genre. Some online music streaming websites offer recommendations to meet the user's demand, for example, Spotify, Pandora, iTunes, etc [3].

\section{Literature Survey}

The Internet is a source of infinite information with rapid growth. There are millions of e-commerce websites on the internet and so are the products available, which leads customers not to make the right decisions. The different components of recommendation systems are items, users and user-item matching algorithms, various approaches of recommendation systems.

The introduction of recommendation systems has proved itself to be priceless and is appreciated because of their ability to make customers make the right choices in time. Recommender Systems are software tools that use techniques for providing product suggestions to a user. The hints identify with different decision-making processes, for example, what things to purchase, what music to tune in to, or what online news to peruse. The recommendation system carries out its process by analyzing the user's visit to its website and remembering their choice. This analysis is further used to suggest the user [4].

Recommendations are classified into two types based on the number of users the system suggests to. When the interest of a single user is noted to provide him the suggestion, it is known as a personal recommender system. Since a personal taste cannot be the same for different users, this type of recommenders is used based on the user's taste or preference. Another type of recommender system is called public recommendations or impersonal recommendations. When a system grabs the interests of users on a large scale, basically to make recommendations based on the popularity of the item [5]. 
By the definition of the recommendation system, it is clear that there would be something that captures the user's behavior, and analyzing that behavior would result in future predictions. The thing that provides this capability to a system is said to be "user modeling" or "user profile". User profile or user modeling is the basic unit of every kind of recommendation system. The framework stores data about the user's behavior into the user's profile. The data is about the user's most continuous visits, top quests, and so forth. A famous e-commerce website, Amazon also uses a recommender system to provide suggestions to its customers. It uses the active search recommender which suggests the buyer items which are similar to his previous searches and similarity-wise [6]. This form of recommender uses the technique called user to use collaborative filtering as the algorithm does the searching of items with users having similar patterns of purchasing. There are millions of choices of music available online. A proper method of filtering and prioritizing among them is needed for different users. To decipher such problems, recommender systems play a vital role by providing the results of top data which is dynamically generated using the required methods and data [7].

\section{Proposing system}

To achieve the goal of the paper, the first process is to do enough background study, so the study will be conducted. The whole paper is based on a big amount of music data so that we choose the quantitative research method which is shown in figure 1. For philosophical assumption, positivism is selected because the paper is experimental and testing character. An approach is a deductive approach as the improvement of our research will be tested by deducing and testing a theory. Ex post facto research is our research strategy, the music data is already collected and we don't change the independent variables. We use experiments to collect music data. Computational mathematics is used in data analysis because the result is based on the improvement of algorithms. For quality assurance, we have a detailed explanation of algorithms to ensure test validity. Similar results will be generated when we run the same data multiple times, which is for reliability [8]. We ensure the same data leading to the same result by different algorithms.

- Popularity algorithm

- Collaborative filtering recommendation

1. User-based collaborative filtering

2. Item-based collaborative filtering

\section{FLOWCHART}

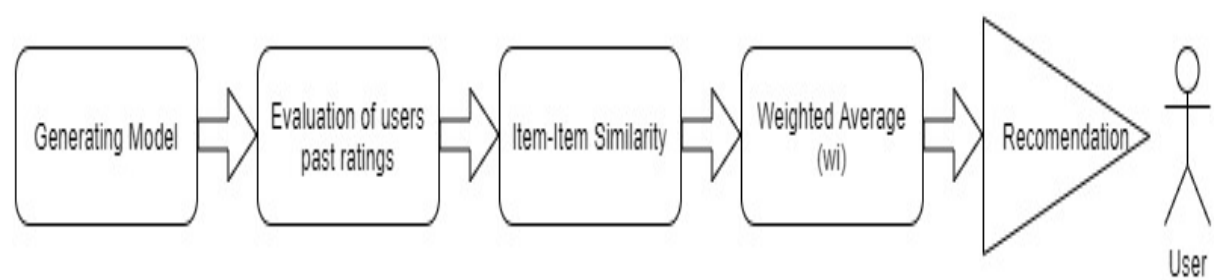

Figure 1. Control flow chart for Proposed model. 
We model the dataset and using Hadoopsten's model of item-based collective filtering (using Hadoi's model). We focus our predictions on users' previous ratings when we use this CF. Another approach to determine item similarities is to build a unititem matrix by utilizing the data already in the system. To sum up, more wood was needed to finish the staircase than to make the tree and its surroundings as spectacular as it was anticipated, yet more scenery was needed to make the surroundings look acceptable as it did." The similarity coefficient (competition coefficient) is used for the estimation. It is the ratio of the intersecting set to the union set of objects or variables taken in the relationship as given in equation 1 . When any user rates an item, the top $n$ similar items are derived from the similarity matrix which is recommended.

$$
T(\mathrm{x}, \mathrm{y})=\frac{N z}{(N x+N y-N z)}
$$

Where $\mathrm{N}_{\mathrm{z}}$ is the No. of Attributes in the intersection set and $\mathrm{N}_{\mathrm{x}}$ and $\mathrm{N}_{\mathrm{y}}$ is No. of Attributes in object $\mathrm{X}$ and $\mathrm{Y}$ respectively.

\section{Approaches in Recommendation System}

\subsection{Popularity model:}

So far, the audience or crowd are reliable with their knowledge contributes to the majority of new business expansion strategies' advice and feedback, which are strong overall. Leveraging content is the core concept of a recommender framework to rank and sort long-tail users' particular preferences, in a straightforward way is rather than serving the objects with simple precision [9]. One limitation of the effective recommendation filtering for active filters is "popularity". This problem originated with the Long Tail phenomenon, which states that a large number of users use very few but popular items while a small number of users consume less popular items. Since collaborative filtering is based on the preferences of people to produce recommendations, it leads to poor variations of recommendations (since most people prefer to use only popular items). E.g. Celma has shown that the music industry follows a long tail [10]. This algorithm isn't personalized; it simply recommends the most popular items to a user. As the popularity is based upon the people count hence it provides better results. The final motive of a system is to provide the best recommendation based on the available features that are both user data and song data.

\subsection{Collaborative filtering recommendation:}

It is a very common method not only for music recommendations but also for other types of recommendation systems. This method relies on a given user content (ratings or full response), and based on the "word of mouth" process of recommended content is recommended by the user if liked by the same users. Because of this, filtering systems do not need to deal with content, which means they do not support the decision to recommend something or not in the description, or the physical properties of an item 
[11]. In the case of music recommendation, it allows avoiding the task of analyzing and categorizing music content. This is an important advantage, given the complexity of music signal analysis and music metadata. And this can be done in two ways collaborative and content-based filtering which shown in figure 2.[12].

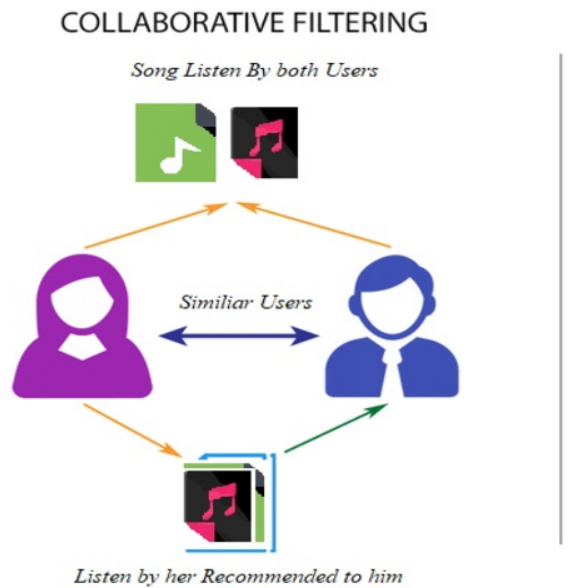

\section{CONTENT-BASED FILTERING}

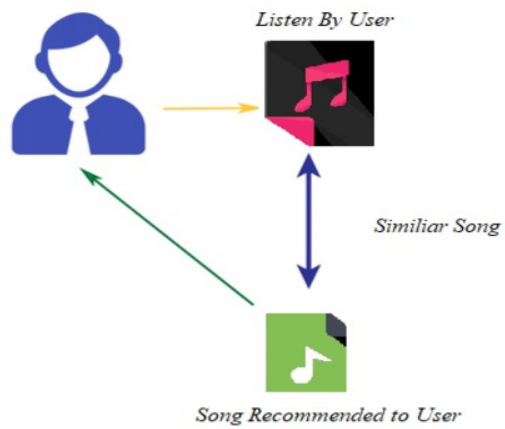

Figure 2. Shows the difference between $\mathrm{CF}$ and C-BF.

\subsection{User-Based Collaborative Filtering:}

User-based filtering is different, it takes into consideration of user-liked items, and depending upon that it predicts better results. The first step of item-based filtering is to calculate items that are liked by the users so they can be recommended to other users. The core point of item-based filtering is to calculate the matching of items. In collaborative filtering, the users are considered similar when they like similar items.

In collaborative filtering, the users are considered similar when they like similar items in Equations 2 and 3 and Table 1.

$$
S_{U, V}=|N(u) \cap N(v)||N(u) \cup N(v)| \quad \mathrm{E} . \mathrm{q}(2)
$$

There are a lot of similarity algorithms, formulas

$$
P_{u i}=\sum_{v \in S(u, k) \cap N(i)} S_{u, v} R_{v, i}
$$

Table 1. an example of a User-based CF recommendation. According to the interesting history of User A, only User C can be the neighbor him, so Item $\mathrm{D}$ will be recommended to User A.

\begin{tabular}{|c|c|c|c|c|}
\hline User/Item & Item-1 & Item-2 & Item-3 & Item-4 \\
\hline User-A & Yes & & Yes & Recommend \\
\hline User-B & & Yes & & \\
\hline User-C & Yes & & Yes & Yes \\
\hline
\end{tabular}




\subsection{Item-Based collaborative filtering:}

Item-based filtering is different, assuming users will like the same things that user preferences do. So, the first step in item-based filtering is to find things like what the user liked before. The main point of item-based filtering is to calculate the similarity of two items. The CF items consider that users' preferences are the same. Item CF considers that items that are liked by more same users, the more similar they are. Assume N(i) and N(j) are user sets who like $i$ and $j$ respectively. Hence the similarity of $i$ and $j$ can be defined as in equations 3 and 4 and Table 2 .

$$
\begin{array}{ll}
S_{i, j}=|N(i) \cap N(j)||N(i) \cup N(j)| & \text { E.q (3). } \\
P_{u i}=\sum_{j \in S(i, k) \cap N(u)} S_{i, j} R_{u, j} & \text { E.q (4). }
\end{array}
$$

While User C prefers Item 1, so we can find that maybe User C loves Item C too.

Table 2. Item-based CF

\begin{tabular}{|c|c|c|c|}
\hline User/Item & Item-1 & Item-2 & Item-3 \\
\hline User-A & Yes & & Yes \\
\hline User-B & Yes & Yes & Yes \\
\hline User-C & Yes & & Recommend \\
\hline
\end{tabular}

\section{Results and Discussion}

The best algorithm is given by the line which occupies much space in the graph when the two algorithms collaborative and popularity are taken into consideration. From the results of both popularity and collaborative algorithms, we can observe that collaboration gives high efficiency when compared to popularity. Hence the collaborative filtering algorithm recommends well. The popularity curve is represented by blue color and the collaborative curve is represented by an orange curve, high the curve good is the performance. As the orange curve is more the performance of collaboration is efficient when compared to popularity algorithms. Which was shown in Table 3.

Table 3. Comparison between popularity algorithm and collaborative algorithm

\begin{tabular}{|c|c|c|}
\hline Model & Precision & Recall \\
\hline Popularity algorithm & 0.87 & 0.83 \\
\hline $\begin{array}{c}\text { Item- Based Collaborative } \\
\text { filtering algorithm }\end{array}$ & 0.90 & 0.86 \\
\hline $\begin{array}{c}\text { User-Based Collaborative } \\
\text { filtering algorithm }\end{array}$ & 0.92 & 0.88 \\
\hline
\end{tabular}




\section{Conclusion}

Understand how the recommendation works in the case of songs and provides the users with the best results. The final result made us understand how to use the data to get a good recommendation output. Furthermore, the results made us understand that good precision and recall from our work improved the overall result. Finally, few methods have been developed that work for providing the users with the best results using details like listen, count, user id, song - artist. Recommendation programs have proven to be the best solution to solve the problem of information overload. Decisions can be made more quickly and easily by the use of time and resources saved Research on music, human behavior, and how it is linked to the impact of music has risen over the last few years, and in particular, to the expansion of the temporal lobe has been active over the last decade Since music is so vital to our daily lives, now that we have greater advancements in technology, we can connect with people anywhere people, more easily. It is very difficult to satisfy the requirements of one's interests and maintain the service in the long term while serving clients are so diverse. Therefore, prospective recommendation systems can enable decisions that are made more intuitive to the consumer, so that they can make the best decisions possible. And of course, it will provide automatic music suggestions to bootleg copies of the song results, which will please the consumer as well. In this paper, we have described the elements of the music recommendation system and the various models that can be used for recommendation such as popularity, collaborative item filtering, user-based filtering. The cooperative complementary model has achieved great success but has issues such as willpower, human effort, etc. Future work will focus on expanding the existing methods and algorithms used so that the forecast recommendation system and the quality of the recommendations can be improved.

\section{Reference}

[1]. Liang, D., Zhan, M., \& Ellis, D. P. (2015, October). Content-Aware Collaborative Music Recommendation Using Pre-trained Neural Networks. In ISMIR pp. 295-301.

[2]. Thota, J.R., Kothuru, M., Shanmuk Srinivas, A., Jitendra M, S.N.V. (2020) “ Monitoring diabetes occurrence probability using classification technique with a UI" International Journal of Scientific and Technology Research, 9 (4): 38-41.

[3]. Amiripalli, S.S., Venkatarao, R., Jitendra, M.S.N.V., Mycherla, N.M.J. (2020) "Detecting emotions of student and assessing the performance by using deep learning" International Journal of Advanced Trends in Computer Science and Engineering, 9 (2): 1641-1645.

[4]. Potharaju, S. P., \& Sreedevi, M. (2017). A Novel Clustering Based Candidate Feature Selection Framework Using Correlation Coefficient for Improving Classification Performance. Journal of Engineering Science \& Technology Review, 10(6).

[5]. Jitendra, M.S.N.V., Radhika, Y. (2020). "A review: Music feature extraction from an audio signal" International Journal of Advanced Trends in Computer Science and Engineering, 9 (2): 973-980.

[6]. Xing, Z., Wang, X., \& Wang, Y. (2014, October). Enhancing Collaborative Filtering Music Recommendation by Balancing Exploration and Exploitation. In Ismir : 445-450

[7]. Wang, H., Wang, N., \& Yeung, D. Y. (2015, August). Collaborative deep learning for recommender systems. In Proceedings of the 21th ACM SIGKDD international conference on knowledge discovery and data mining : 1235-1244).

[8]. Amiripalli, S. S., \& Bobba, V. (2020). An Optimal Graph based ZigBee Mesh for Smart Homes. Journal of Scientific \& Industrial Research Vol. 79(4): 318-322.

[9]. Kavinkumar, V., Reddy, R. R., Balasubramanian, R., Sridhar, M., Sridharan, K., \& Venkataraman, D. (2015, August). A hybrid approach for recommendation system with added feedback component. In 2015 International Conference on Advances in Computing, Communications and Informatics (ICACCI) : 745-752. IEEE. 
[10]. Sunitha, M., \& Adilakshmi, T. (2018). Music Recommendation System with User-Based and Item-Based Collaborative Filtering Technique. In Networking Communication and Data Knowledge Engineering (pp. 267-278). Springer, Singapore.

[11]. Mukkamala S.N.V. Jitendra and Y. Radhika, "Singer Gender Classification using Feature-based and Spectrograms with Deep Convolutional Neural Network" International Journal of Advanced $\begin{array}{lllll}\text { Computer Science and } & \text { Applications }\end{array}$ http://dx.doi.org/10.14569/IJACSA.2021.0120218.

[12]. Potharaju, S. P., Sreedevi, M., Ande, V. K., \& Tirandasu, R. K. (2019). Data mining approach for accelerating the classification accuracy of cardiotocography. Clinical Epidemiology and Global Health, 7(2), 160-164. 